\title{
AUTHOR INDEX \\ VOLUME 8 (1994)
}

Abe, N., see Kakusho, K.

Adda, G., see Gauvain, J.L.

Agarwal, A., see Mui, L.

Aggarwal, J.K., see Chaudhary, V.

Ahuja, N., see Hu, X.-P.

Aizawa, K. \& Nakamura, A., Path-controlled graph grammars for syntactic pattern recognition

Alpaydin, E., GAL: Networks that grow when they learn and shrink when they forget

Arunachalam, P., see Chaudhary, V.

Baggia, P., Fissore, L., Giachin, E., Micca, G., Rullent, C. \& Laface, $P$., A speech understanding system for information retrieval

Bagherzadeh, N., see Kavianpour, A.

Baird, H., see Bunke, $H$.

Baird, H.S., Background structure in document images

Beaumier, L., see Sabourin, R.

Bergevin, R., see Hébert, $\mathrm{P}$.

Binaghi, E., Gagliardi, I. \& Schettini, R., Image retrieval using fuzzy evaluation of color similarity

Boyer, K.L., Stark, L. \& Bunke, H., Applications of artificial intelligence, machine vision, and robotics

Brady, M., see Du, F.-L.

Brittan, P., see Fairhurst, M.C.

Buehrer, D.J., see Chang, C.-C.

Bunke, H., see Boyer, K.L.

Bunke, H., see Ha, T.M.

Bunke, H., Wang, P.S.P. \& Baird, H., Document image analysis

Cardot, H., Revenu, M., Victorri, B. \& Revillet, M.-J., A static signature verification system based on a cooperating neural networks architecture

Casacuberta, F., see Galiano, I.

Chang, C.-C., Chen, Y.-W. \& Buehrer, D.J., A two-dimensional shape recognition scheme based on principal component analysis

Chang, H.-D. \& Wang, J.-F., A robust stroke extraction method for handwritten Chinese characters

Chang, S.-C., see Wang, M.-J.J.

Charot, F., see Frison, P.

Chaudhary, V., Kumari, K., Arunachalam, P. \& Aggarwal, J.K., Manipulations of octrees and quadtrees on multiprocessors

Chen, C.-H., see Lee, J.-D.

Chen, S.-H., see Wang, Y.-R.
8 (1994) $577-593$

8 (1994) $99-131$

8 (1994) 1189-1204

8 (1994) 439-455

8 (1994) 1343-1379

8 (1994) 485-500

8 (1994) 391-414

8 (1994) 439-455

8 (1994) 71-97

8 (1994) 337-349

8 (1994) 997

8 (1994) 1013-1030

8 (1994) 709-748

8 (1994) 1319-1342

8 (1994) 945-968

8 (1994) 1269-1271

8 (1994) 1439-1469

8 (1994) 661-678

8 (1994) 859-875

8 (1994) 1269-1271

8 (1994) 1053-1080

8 (1994) 997

8 (1994) 679-692

8 (1994) 155-180

8 (1994) 859-875

8 (1994) 1223-1239

8 (1994) 899-917

8 (1994) 417-438

8 (1994) 439-455

8 (1994) 351-371

8 (1994) 233-246 
Chen, Y.-W., see Chang, C.-C.

8 (1994) 859-875

Chen, Z. \& Wang, C.-M., A two-parameter generate-and-test method for camera parameter estimation with any planar calibration object

8 (1994) $877-898$

Chou, W., Lee, C.-H., Juang, B.-H. \& Soong, F.K., A minimum error rate pattern recognition approach to speech recognition

Collin, S. \& Colnet, D., Syntactic analysis of technical drawing dimensions

Colnet, D., see Collin, S.

Dan, S., see Kakusho, K.

Dare, V.R., see Siromoney, R.

Dimauro, G., Impedovo, S. \& Pirlo, G., Component-oriented algorithms for signature verification

Du, F.-L. \& Brady, M., A four degree-of-freedom robot head for active vision

Endo, M., see Makino, S.

Essen, U., see Ney, H.

Fairhurst, M.C. \& Brittan, P., An evaluation of parallel strategies for feature vector construction in automatic signature verification systems

Fissore, L., see Baggia, P.

Fohr, D., Haton, J.-P. \& Laprie, Y., Knowledge-based techniques in acoustic-phonetic decoding of speech: interest and limitations

Frison, P., Charot, F., Gautrin, E., Lavenier, D., Quinton, P., Raimbault, F. \& Wagner, C., From equations to hardware. Towards the systematic mapping of algorithms onto parallel architectures

8 (1994) $5-31$

8 (1994) 1131-1148

8 (1994) 1131-1148

8 (1994) $577-593$

8 (1994) 627-639

8 (1994) 771-793

8 (1994) 1439-1469

8 (1994) $197-213$

8 (1994) 33-70

8 (1994) 661-678

8 (1994) 71-97

8 (1994) 133-153

Furui, S., see Shikano, K.

Gagliardi, I., see Binaghi, E.

Galiano, I., Sanchis, E., Casacuberta, F. \& Torres, I., Acoustic-phonetic decoding of Spanish continuous speech

Gautrin, E., see Frison, P.

Gauvain, J.L., Lamel, L.F., Adda, G. \& Mariani, J., Speech-to-text conversion in French

Giachin, E., see Baggia, P.

Goh, W.L., see Mital, D.P.

Gregor, J., Data-driven inductive inference of finite-state automata

Grewe, L. \& Kak, A., Integration of geometric and non-geometric attributes for fast object recognition

Gupta, A., see Mui, L.

Ha, T.M. \& Bunke, H., Model-based analysis and understanding of check forms

Haeb-Umbach, R., see Ney, H.

Hamamoto, Y., see Kobara, K.

Hanazawa, T., see Shikano, $K$.

Hastie, T., see Nelson, W.

Haton, J.-P., see Fohr, D.
8 (1994) 417-438
8 (1994) 181-196
8 (1994) 945-968
8 (1994) 155-180
8 (1994) 417-438
8 (1994) $99-131$
8 (1994) 71-97
8 (1994) 845-857
8 (1994) 305-322
8 (1994) 1407-1437
8 (1994) 1189-1204
8 (1994) 1053-1080
8 (1994) 33-70
8 (1994) 595-607
8 (1994) 181-196
8 (1994) 749-770
8 (1994) 133-153 
Hébert, P., Laurendeau, D. \& Bergevin, R., From 3-D scattered data to geometric signal description: Invariant stable recovery of straight line segments

Holeva, L.-F., Range estimation from camera blur by regularized adaptive identification

Hosokawa, K., see Yamada, H.

Hu, X.-P. \& Ahuja, N., Feature extraction and matching as signal detection

Hughes, K. \& Ranganathan, N., Modeling sensor confidence for sensor integration tasks

Hwang, T.-F., see Tseng, J.C.R.

Impedovo, S., see Dimauro, G.

Inagaki, Y., see Ito, A.

Inoue, K., see Ito, A.

Inoue, K. \& Takanami, I., A characterization of recognizable picture languages

Inoue, K., Nakamura, A., Nivat, M., Saoudi, A. \& Wang, P.S.P., Parallel image analysis and processing

Ito, A., Inoue, K., Takanami, I. \& Inagaki, Y., Constant leaf-size hierarchy of two-dimensional alternating turing machines

Ito, A., see Makino, S.

Juang, B.-H., see Chou, W.

Juang, B.-H., see Lee, L.-S.

Kak, A., see Grewe, L.

Kakusho, K., Dan, S., Abe, N. \& Kitahashi, T., Shape recovery and error correction based on hypothetical constraints by parallel network for energy minimization

Kanai, J., see Rice, S.V.

Kanaoka, T., see Kobara, K.

Kavianpour, A. \& Bagherzadeh, N., Parallel algorithms for

line detection on a pyramid architecture

Kawabata, T., see Shikano, K.

Kido, K., see Makino, S.

Kim, H.S., see Koo, J.M.

Kim, J.H., see Kim S.H.

Kim, S.H. \& Kim, J.H., Automatic input of logic diagrams by recognizing loop-symbols and rectilinear connections

Kitahashi, T., see Kakusho, K.

Kobara, K., Kanaoka, T., Tomita, S., Hamamoto, Y. \& Munechika, K., Use of gradated patterns in an associative neural memory for invariant pattern recognition

Kodratoff, Y. \& Moscatelli, S., Machine learning for object recognition and scene analysis

Koo, J.M., Kim, H.S. \& Un, C.K., A Korean large vocabulary speech recognition system for automatic telephone number query service

Kumari, K., see Chaudhary, V.

Laface, P., see Baggia, P.

Lamel, L.F., see Gauvain, J.L.

Laprie, Y., see Fohr, D.
8 (1994) 1319-1342

8 (1994) 1273-1300

8 (1994) 1149-1170

8 (1994) 1343-1379

8 (1994) 1301-1318

8 (1994) 919-944

8 (1994) 771-793

8 (1994) 509-524

8 (1994) 509-524

8 (1994) 501-508

8 (1994) 415-416

8 (1994) 509-524

8 (1994) 197-213

8 (1994) 5-31

8 (1994) 1-3

8 (1994) 1407-1437

8 (1994) 577-593

8 (1994) 1259-1268

8 (1994) 595-607

8 (1994) 337-349

8 (1994) 181-196

8 (1994) 197-213

8 (1994) 215-232

8 (1994) 1113-1129

8 (1994) 1113-1129

8 (1994) 577-593

8 (1994) 595-607

8 (1994) 259-304

8 (1994) 215-232

8 (1994) 439-455

8 (1994) 71-97

8 (1994) 99-131

8 (1994) 133-153 
Laroche, P., Nivat, M. \& Saoudi, A., Context-sensitivity of puzzle grammars

Laurendeau, D., see Hébert, P.

Lavenier, D., see Frison, P.

Leclerc, F. \& Plamondon, R., Automatic signature verification:

The state of the art - 1989-1993

Lee, C.-H., see Chou, W.

Lee, J.-D., Lee, J.-Y., You, Y.-C. \& Chen, C.-H., Determining location and orientation of a labelled cylinder using point-pair estimation algorithm

Lee, J.-Y., see Lee, J.-D.

Lee, L.-S. \& Juang, B.-H., On speech recognition for different languages

Li, T.F. \& Yu, S.S., Handprinted Chinese character recognition using probability distribution feature

Li, T.F., Unsupervised learning of prior weights for Bayes rules in pattern recognition

Lim, A.W.T., see Mital, D.P.

Liu, C.-M., see Wang, M.-J.J.

Makino, S., Ito, A., Endo, M. \& Kido, K., A continuous speech recognition system using a modified LVQ2 method and a dependency grammar with semantic constraints

8 (1994) $525-542$
8 (1994) $1319-1342$
8 (1994) $417-438$

8 (1994) 643-660

8 (1994) $5-31$

8 (1994) 351-371

8 (1994) 351-371

8 (1994) $1-3$

8 (1994) 1241-1258

8 (1994) 323-335

8 (1994) 969-995

8 (1994) 899-917

8 (1994) 197-213

8 (1994) 99-131

8 (1994) 627-639

8 (1994) 181-196

8 (1994) 71-97

8 (1994) 465-483

8 (1994) $845-857$ texture analysis

Mital, D.P., Teoh, E.K. \& Lim, A.W.T., A hybrid method towards the segmentation of range images for 3-D object recognition

Mori, S., see Nishida, H.

Morita, K. \& Ueno, S., Parallel generation and parsing of array languages using reversible cellular automata

Moscatelli, S., see Kodratoff, Y.

Mui, L., Agarwal, A., Gupta, A. \& Wang, P.S.P., An adaptive modular neural network with application to unconstrained character recognition

Munechika, K., see Kobara, K.

Nakamura, A., see Aizawa, K.

Nakamura, A., see Inoue, K.

Nartker, T.A., see Rice, S.V.

Nelson, W., Turin, W. \& Hastie, T., Statistical methods for on-line signature verification

Ney, H., Steinbiss, V., Haeb-Umbach, R., Tran, B.-H. \&

Essen, U., An Overview of the Philips research system for large-vocabulary continuous speech recognition

Nishida, H. \& Mori, S., A model-based split-and-merge method for character string recognition

Nivat, M., see Inoue, $\mathrm{K}$.
8 (1994) 969-995

8 (1994) 1205-1222

8 (1994) 543-561

8 (1994) 259-304

8 (1994) 1189-1204

8 (1994) 595-607

8 (1994) 485-500

8 (1994) 415-416

8 (1994) 1259-1268

8 (1994) 749-770

8 (1994) 33-70

8 (1994) 1205-1222

8 (1994) 415-416 
Nivat, M., see Laroche, P.

Nivat, M., see Saoudi, A.

Pai, H.-F., see Wang, H.-C.

Papamarkos, N., Spiliotis, I. \& Zoumadakis, A., Character recognition by signature approximation

Pinhanez, C.S., Behavior-based active vision

Pirlo, G., see Dimauro, G.

Plamondon, R., Automatic signature verification

Plamondon, R., see Leclerc, F.

Plamondon, R, see Sabourin, R.

Plamondon, $R$, The design of an on-line signature verification system: from theory to practice

Quinton, P., see Frison, $P$.

Raimbault, F., see Frison, $P$.

Ranganathan, N. \& Sastry, R., VLSI architectures for pattern matching

Ranganathan, N., see Hughes, $\mathrm{K}$.

Revenu, M., see Cardot, $\mathrm{H}$.

Revillet, M.-J., see Cardot, H.

Rice, S.V., Kanai, J. \& Nartker, T.A., An algorithm for matching OCR-generated text strings

Rosin, P.L., Non-parametric multiscale curve smoothing

Rullent, C., see Baggia, P.

Sabourin, R., Plamondon, R. \& Beaumier, L., Structural interpretation of handwritten signature images

Saito, T., see Yamada, H.

Sanchis, E., see Galiano, I.

Sandon, P.A., Control of eye and arm movements using active, attentional vision

Saoudi, A. \& Nivat, M., Parallel algorithms for multi-dimensional image template matching

Saoudi, A., see Inoue, $\mathrm{K}$.

Saoudi, A., see Laroche, $\mathrm{P}$.

Sastry, R., see Ranganathan, N.

Schettini, R., see Binaghi, E.

Shieh, J.-M., see Wang, Y.-R.

Shikano, K., Yamada, T., Kawabata, T., Matsunaga, S., Furui, S \& Hanazawa, T., Dictation machine based on Japanese character source modeling

Siromoney, R., Mathew, L., Subramanian, K.G. \& Dare, V.R.,

Learning of recognizable picture languages

Soong, F.K., see Chou, W.

Spiliotis, I., see Papamarkos, N.

Srihari, S.N., see Wang, D.

Stark, L., see Boyer, K.L.

Steinbiss, V., see Ney, H.

Subramanian, K.G., see Siromoney, R.

Suen, C.Y., see Tang, Y.Y.

Takada, Y., Learning equal matrix grammars based on control sets

Takanami, I., see Inoue, K.
8 (1994) 525-542

8 (1994) 457-464

8 (1994) 247-257

8 (1994) 1171-1187

8 (1994) 1493-1526

8 (1994) 771-793

8 (1994) 641-642

8 (1994) 643-660

8 (1994) $709-748$

8 (1994) 795-811

8 (1994) 417-438

8 (1994) $417-438$

8 (1994) 815-843

8 (1994) 1301-1318

8 (1994) 679-692

8 (1994) 679-692

8 (1994) 1259-1268

8 (1994) 1381-1406

8 (1994) 71-97

8 (1994) 709-748

8 (1994) 1149-1170

8 (1994) 155-180

8 (1994) 1471-1491

8 (1994) 457-464

8 (1994) 415-416

8 (1994) 525-542

8 (1994) 815-843

8 (1994) 945-968

8 (1994) 233-246

8 (1994) 181-196

8 (1994) 627-639

8 (1994) 5-31

8 (1994) 1171-1187

8 (1994) 1031-1052

8 (1994) 1269-1271

8 (1994) 33-70

8 (1994) 627-639

8 (1994) 1081-1111

8 (1994) 609-626

8 (1994) 501-508 
Takanami, I., see Ito, A.

Tang, Y.Y. \& Suen, C.Y., Document structures: A survey

Tanimoto, S.L. \& Miller, R., Mesh algorithms for finding repetitions and partial symmetries in arrays

Teoh, E.K., see Mital, D.P.

Tomita, S., see Kobara, K.

Torres, I., see Galiano, I.

Tran, B.-H., see Ney, H.

Tseng, J.C.R., Hwang, T.-F. \& Yang, W.-P., Efficient image retrieval algorithms for large spatial databases

Turin, W., see Nelson, W.

Ueno, S., see Morita, K.

Un, C.K., see Koo, J.M.

Victorri, B., see Cardot, H.

Wagner, C., see Frison, $\mathrm{P}$.

Wang, C.-M., see Chen, Z.

Wang, D. \& Srihari, S.N., Analysis of form images

Wang, H.-C. \& Pai, H.-F., Recognition of Mandarin syllables based on the distribution of two-dimensional cepstral coefficients

Wang, J.-F., see Chang, H.-D.

Wang, M.-J.J., Chang, S.-C., Liu, C.-M. \& Wu, W.Y., A new edge detection method through template matching

Wang, P.S.P., see Bunke, $\mathrm{H}$.

Wang, P.S.P., see Inoue, $\mathrm{K}$.

Wang, P.S.P., see Mui, L.

Wang, P.S.P., see Zhang, Y.Y.

Wang, P.S.P., Three-dimensional sequential/parallel universal array grammars for polyhedral object pattern analysis

Wang, T., Learning of the Hopfield associative memory by global minimization

Wang, Y.-R., Shieh, J.-M. \& Chen, S.-H., Tone recognition of continuous Mandarin speech based on hidden Markov model

Wu, W.Y., see Wang, M.-J.J.

Yamada, H., Yamamoto, K., Saito, T. \& Hosokawa, K., Recognition of elevation value in topographic maps by multi-angled parallelism

Yamada, T., see Shikano, K.

Yamamoto, K., see Yamada, $\mathrm{H}$.

Yang, W.-P., see Tseng, J.C.R.

Yoshimura, I. \& Yoshimura, M., Off-line verification of Japanese signatures after elimination of background patterns

Yoshimura, M., see Yoshimura, I.

You, Y.-C., see Lee, J.-D.

Yu, S.S., see Li, T.F.

Zhang, Y.Y. \& Wang, P.S.P., A new parallel thinning algorithm

Zoumadakis, A., see Papamarkos, N.
8 (1994) 509-524

8 (1994) 1081-1111

8 (1994) 465-483

8 (1994) 969-995

8 (1994) 595-607

8 (1994) 155-180

8 (1994) 33-70

8 (1994) 919-944

8 (1994) 749-770

8 (1994) 543-561

8 (1994) 215-232

8 (1994) 679-692

8 (1994) 417-438

8 (1994) 877-898

8 (1994) 1031-1052

8 (1994) 247-257

8 (1994) 1223-1239

8 (1994) 899-917

8 (1994) 997

8 (1994) 415-416

8 (1994) 1189-1204

8 (1994) 999-1011

8 (1994) 563-576

8 (1994) 373-390

8 (1994) 233-246

8 (1994) 899-917

8 (1994) 1149-1170

8 (1994) 181-196

8 (1994) 1149-1170

8 (1994) 919-944

8 (1994) 693-708

8 (1994) 693-708

8 (1994) 351-371

8 (1994) 1241-1258

8 (1994) 999-1011

8 (1994) 1171-1187 